\title{
Drug Resistance in Filarial Parasites Does Not Affect Mosquito Vectorial Capacity
}

\author{
Erik Neff ${ }^{1, *(1)}$, Christopher C. Evans ${ }^{2}$, Pablo D. Jimenez Castro ${ }^{2,3} \mathbb{C}$, Ray M. Kaplan ${ }^{2}$ and Guha Dharmarajan $1, * \mathbb{C}$ \\ 1 Savannah River Ecology Laboratory, University of Georgia, Drawer E, Aiken, SC 29802, USA \\ 2 Department of Infectious Diseases, College of Veterinary Medicine, University of Georgia, \\ Athens, GA 30602, USA; ccevans@uga.edu (C.C.E.); pablo.jimenezca25@uga.edu (P.D.J.C.); \\ rkaplan@uga.edu (R.M.K.) \\ 3 Grupo de Parasitología Veterinaria, Universidad Nacional de Colombia, Bogotá 11001000, Colombia \\ * Correspondence: eneff93@gmail.com (E.N.); guha@srel.uga.edu (G.D.)
}

Citation: Neff, E.; Evans, C.C.; Jimenez Castro, P.D.; Kaplan, R.M.; Dharmarajan, G. Drug Resistance in Filarial Parasites Does Not Affect Mosquito Vectorial Capacity. Pathogens 2021, 10, 2. https://dx.doi.org/ $10.3390 /$ pathogens 10010002

Received: 15 November 2020 Accepted: 18 December 2020 Published: 22 December 2020

Publisher's Note: MDPI stays neutral with regard to jurisdictional claims in published maps and institutional affiliations.

Copyright: (c) 2020 by the authors. Licensee MDPI, Basel, Switzerland. This article is an open access article distributed under the terms and conditions of the Creative Commons Attribution (CC BY) license (https: / / creativecommons.org/ licenses/by/4.0/).

\begin{abstract}
Parasite drug resistance presents a major obstacle to controlling and eliminating vectorborne diseases affecting humans and animals. While vector-borne disease dynamics are affected by factors related to parasite, vertebrate host and vector, research on drug resistance in filarial parasites has primarily focused on the parasite and vertebrate host, rather than the mosquito. However, we expect that the physiological costs associated with drug resistance would reduce the fitness of drug-resistant vs. drug-susceptible parasites in the mosquito wherein parasites are not exposed to drugs. Here we test this hypothesis using four isolates of the dog heartworm (Dirofilaria immitis) - two drug susceptible and two drug resistant-and two vectors-the yellow fever mosquito (Aedes aegypti) and the Asian tiger mosquito (Ae. albopictus) - as our model system. Our data indicated that while vector species had a significant effect on vectorial capacity, there was no significant difference in the vectorial capacity of mosquitoes infected with drug-resistant vs. drug-susceptible parasites. Consequently, contrary to expectations, our data indicate that drug resistance in D. immitis does not appear to reduce the transmission efficiency of these parasites, and thus the spread of drugresistant parasites in the vertebrate population is unlikely to be mitigated by reduced fitness in the mosquito vector.
\end{abstract}

Keywords: Aedes spp.; arthropod; disease transmission; Dirofilaria immitis; drug resistance; extrinsic incubation period; filaria; macrocyclic lactones; mosquito; vector competence

\section{Introduction}

Vector-borne diseases remain an important challenge for public health globally, and contribute to over $17 \%$ of the global estimated burden of all infectious diseases [1]. Recent few decades have seen encouraging progress in the control of some important mosquitoborne diseases including malaria [2,3] and filariasis [4]. However, continued success in controlling these diseases faces numerous challenges, with the evolution of resistance against anti-parasitic drugs being one of the most important [5-7].

Given that the evolutionary dynamics of parasites are critically affected by the host environment (e.g., immune response [8]), vector-transmitted parasites are subjected to very different selection pressures in the different hosts (vertebrate and invertebrate) used to complete the life cycles. The differential pressures that parasites face in the vertebrate host vs. the arthropod vector could act to enhance or reduce parasite transmission, and thus impact the spread of drug-resistant parasite isolates. For example, the development of drug resistance in vector-transmitted parasites could have high fitness advantages for life history stages in the vertebrate hosts wherein they are likely to be exposed to chemoprophylaxis. However, drug resistance usually trades off against other fitness parameters (e.g., the ability of parasites to reproduce or evade the immune system), and thus drug-resistant genotypes are expected to have lower fitness in drug-free environments $[9,10]$. Thus, we expect that 
the drug-resistant strains may be less fit than their drug-susceptible counterparts in the vector, since parasites are not exposed to drugs in the mosquito.

If drug resistance trades off against physiological characteristics required by the parasite to infect the mosquito host, passage through the vector will select against drugresistant parasite isolates. Indeed, previous studies in malaria parasites have shown that isolates resistant to the anti-malarial compound atovaquone have increased fitness in humans treated with the drug, but fail to develop, and hence be transmitted by the mosquito vector [11]. While drug resistance is an important factor affecting our ability to control filarial parasites globally, to our knowledge no studies have quantified the effects of drug resistance on the ability of a mosquito to transmit the parasite (i.e., vectorial capacity). In contrast to malaria parasites that generally have low fitness consequences in the natural vectors [12], filarial parasites generally induce high levels of mortality in mosquitoes $[13,14]$. Since vectorial capacity is a product of the number of parasites developing to the infectious stage and the probability of the vector surviving to transmit these stages to the vertebrate host [14], we expect that there will be complex interactions that ultimately determine whether drug resistance increases or decreases vectorial capacity. Consequently, to visualize these expectations, we built a model to simulate the effects of drug resistance and susceptibility on vectorial capacity (see Section 4 for details).

Our simulation model revealed that in the case of filarial parasites, the vectorial capacity of mosquitoes infected with drug-susceptible vs. drug-resistant parasite isolates would be affected by a complex interaction between three parameters: (a) establishment load: the initial load of establishing parasites; (b) vector efficiency: the proportion of establishing parasites that develop to L3; (c) survival to the extrinsic incubation period (EIP), which is the minimum period of time required for parasites to develop to their infectious stage in the vector (see Figure 1).
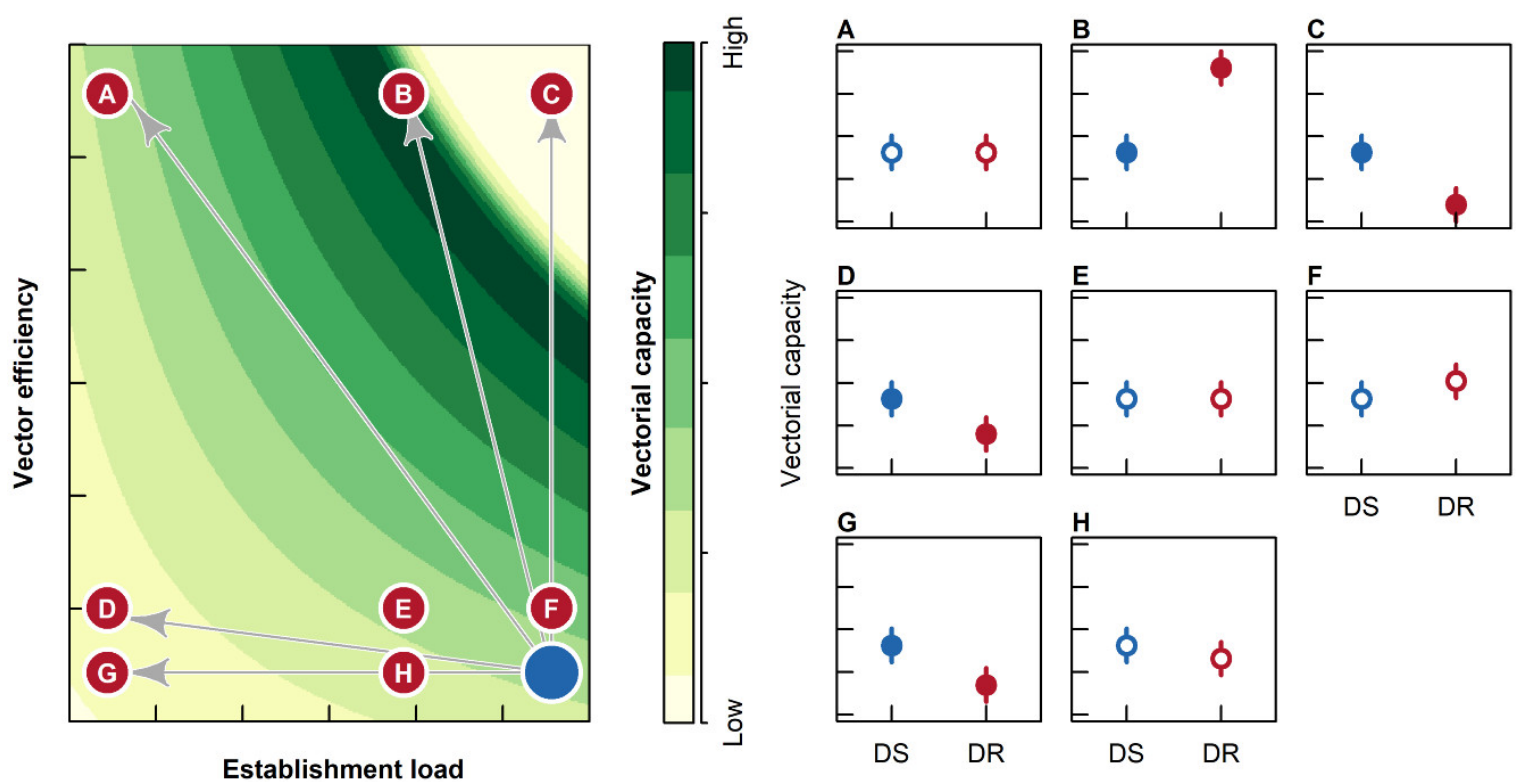

Figure 1. Predicted differences in mosquito vectorial capacity when infected with drug-susceptible (blue symbols) vs. drug-resistant (red symbols) isolates of $D$. immitis. The heat map on the left shows the interacting effects of establishment load (i.e., initial load of establishing parasites) and vector efficiency (proportion of establishing parasites that develop to L3) on vectorial capacity. Vectorial capacity was calculated as the combined product of establishment load, vectorial efficiency and probability of survival to the extrinsic incubation period (EIP). The latter was calculated using a logistic function dependent on predicted L3 parasite load at EIP. We assume that drug resistance will lead to a reduction in establishment load (due to reduced parasite fitness) and increased vector efficiency (due to reduced pathogenicity). These parameters interact to produce a complex set of expectations, with either significant (filled symbols) or non-significant (non-filled symbols) differences between vectorial capacity between drug-susceptible (blue symbols) or drug-resistant (red symbols) parasite isolates (A-H; see text for details). 
Specifically, in cases where drug resistance is associated with a large decrease in establishment load (Figure 1A,D,G), there would a significant reduction in vectorial capacity compared to drug-susceptible isolates, except in the case when drug resistance is also associated with a very large increase in vector efficiency (Figure 1A). Alternatively, when drug resistance is associated with a large increase in vector efficiency (but only a small reduction in establishment load), we expect higher vectorial capacity with drugresistant vs. drug-susceptible isolates (Figure 1B). However, when a large increase in vector efficiency is combined with no reduction in establishment load, the high parasite load reduces survival to EIP, and thus is expected to reduce the vectorial capacity of drug-resistant vs. drug-susceptible isolates (Figure 1C). Small reductions in establishment load and/or increases in vector efficiency will have no detectable effects on vector capacity (Figure 1E,F,H). Consequently, contrary to the case of malaria, vectorial capacity is lower in drug-resistant vs. drug-susceptible parasites only under conditions of very low establishment load (Figure 1D,G) or very high vector efficiency (Figure 1C). This latter effect is due to the high fitness costs associated with filarial infection. Alternatively, under most other conditions, drug-resistant strains face few mosquito-related transmission costs (Figure 1A,E,F,H), and could even show higher transmission under some conditions compared to drug-susceptible strains (Figure 1B).

In this study, we focused on elucidating the effects of drug resistance on the vectorial capacity of mosquitoes infected with dog heartworm (Dirofilaria immitis). Dirofilaria immitis is a mosquito-transmitted filarial parasite that primarily infects dogs and other canids $([15,16]$; see Section 4 or detailed life history characteristics). We used four isolates of the dog heartworm that were either susceptible or resistant to macrocyclic lactone anthelmintics (the primary chemotherapeutic approach to prevent $D$. immitis infections in dogs) as our model system. We studied parasite infection dynamics in two species of mosquitoesthe yellow fever mosquito (Ae. aegypti) and the Asian tiger mosquito (Ae. albopictus)that are natural vectors of $D$. immitis $[17,18]$ (see Section 4 for details). Drug resistance against macrocyclic lactones is a complex issue that compromises our ability to control D. immitis infections in dogs globally, and is an issue that is growing concern in the United States [19-22]. While numerous studies have documented the rise in resistance against macrocyclic lactones, the underlying genetic mechanisms driving this trait in $D$. immitis populations remains unclear $[23,24]$. Additionally, we also have little information on the potential fitness consequences associated with the drug-resistant phenotypes especially as it relates to their ability to be transmitted by the mosquito vector. A better understanding of the role that mosquitoes play in facilitating or inhibiting the spread of drug resistance D. immitis isolates during its life cycle has broad implications for developing transmission models and for implementing control strategies for filarial infections globally.

\section{Results}

\subsection{Establishment Load}

We found that despite standardization of $\mathrm{mf}$ concentrations to $5000 \mathrm{mf} / \mathrm{mL}$ in the infected blood used to feed mosquitoes, the initial establishment load differed by parasite isolate and vector isolate, with a significant interaction between these variables (Figure 2A; $\mathrm{N}=118$; parasite isolate: $\chi^{2}=19.772 ; p<0.001$; vector isolate: $\chi^{2}=8.694 ; p=0.003$; parasite isolate $\times$ vector isolate: $\chi^{2}=3.716 ; p=0.294$ ). The most striking effect we observed was that the Yazoo isolate had the lowest establishment load in both mosquito species. However, this seems to be an isolate-specific effect, and contrary to expectation (see Figure 1), there was no overall reduction in establishment load associated with infection by drug-resistant vs. drug-susceptible parasite isolates (Figure 2A). 
A
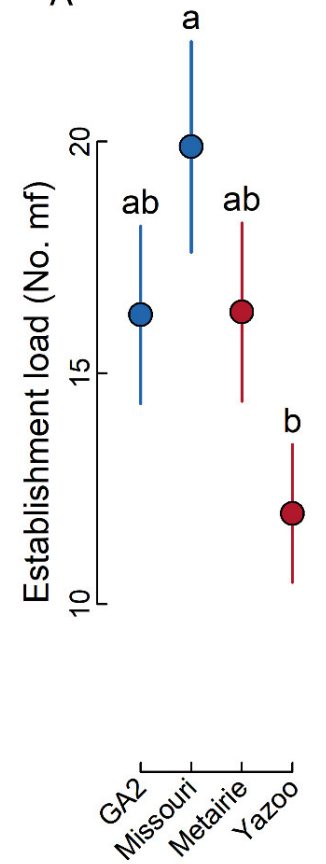

C

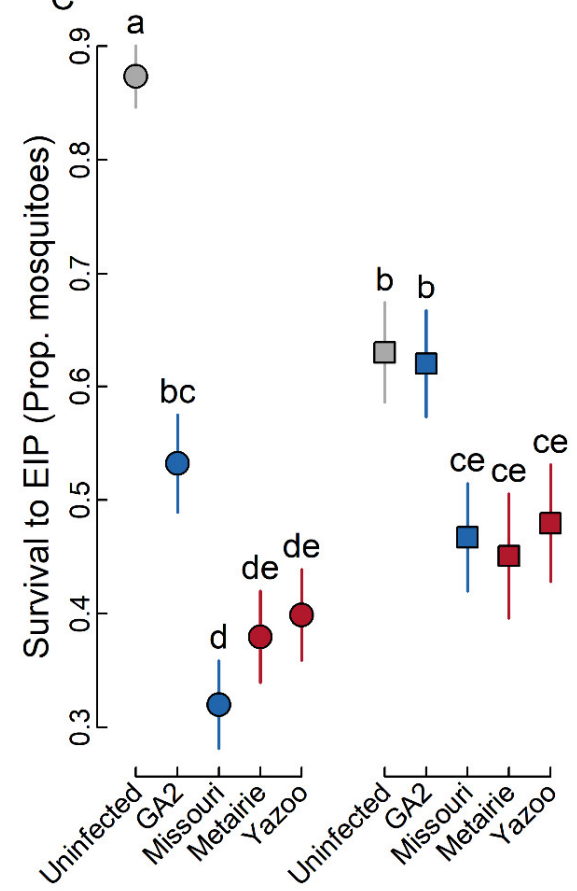

B

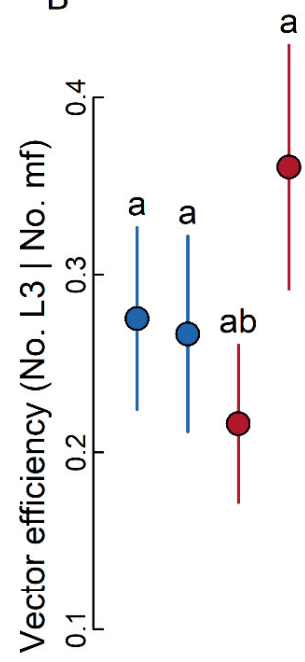

c

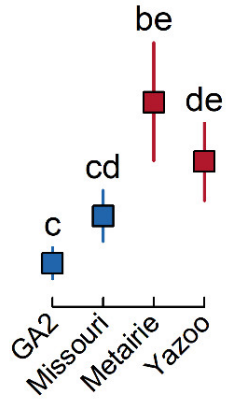

D

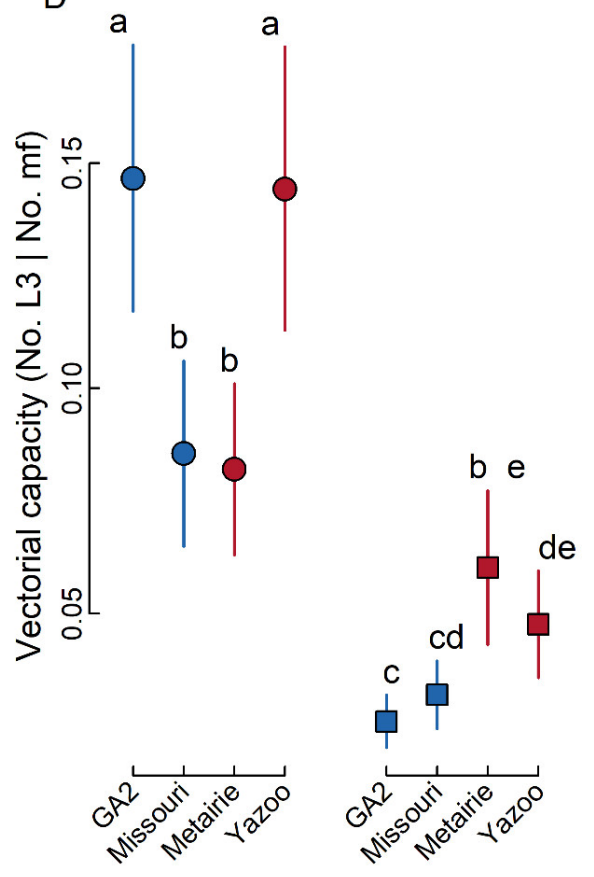

Figure 2. Effects of parasite isolate on parasite transmission risk in Ae. aegypti Liverpool Blackeye (A-D, left side of graphics) and Ae. albopictus (A-D, right side of graphics). Parasite isolates include two drug-susceptible (GA-2 and Missouri; blue symbols) and drug-resistant (Metairie and Yazoo; red symbols) isolates. Separate graphs are given for: (A) establishment load measured as the number of viable microfilaria $(\mathrm{mf})$ counted immediately after infection; (B) vector efficiency measured as the number of infective L3 parasites developing per $\mathrm{mf}$ in mosquitoes surviving to the extrinsic incubation period (EIP); (C) survival to the EIP measured as the proportion of surviving mosquitoes; (D) vectorial capacity measured as the number of infective L3 parasites developing per mf, accounting for the probability of survival to EIP. Least square means standard error are error bars and least square mean values from the GLMER models show significance when they do not share a letter. 


\subsection{Vector Efficiency}

We found that vector efficiency (i.e., proportion of $\mathrm{mf}$ that develop to L3 parasites at EIP) differed by parasite isolate and vector isolate, with a significant interaction between these variables (Figure 2B; $\mathrm{N}=353$; parasite isolate: $\chi^{2}=7.434 ; p=0.059$; vector isolate: $\chi^{2}=77.422 ; p<0.001$; parasite isolate $\times$ vector isolate: $\left.\chi^{2}=10.609 ; p=0.014\right)$. However, again contrary to expectation (see Figure 1), there was no overall difference in vector efficiency between mosquitoes infected with drug-susceptible (i.e., GA-2 and Missouri) vs. drug-resistant (i.e., Metairie and Yazoo) parasite isolates. Vector species plays an important role in vector efficiency, with the Metairie isolate of Ae. albopictus having very low vector efficiency compared to the Liverpool Blackeye isolate of Ae. aegypti (Figure 2B).

\subsection{Survival to EIP}

We found that parasite isolate and vector species interacted strongly to affect mosquito survival to EIP (Figure 2C; $N=1331$; parasite isolate: $\chi^{2}=80.145 ; p<0.001$; vector isolate: $\chi^{2}=1.120 ; p=0.290$; parasite isolate $\times$ vector isolate: $\chi^{2}=31.512 ; p<0.001$ ). It is important to recognize that the term "parasite isolate" in this analysis also included the uninfected controls as one category. Thus, the global effects reported here incorporate both the differences in survival between infected and uninfected mosquitoes, as well as differences in survival between the different parasite isolates. One of the most interesting findings of this analysis was the exceptionally large reduction in survival between infected and uninfected Ae. aegypti mosquitoes (Figure 2C). However, in the case of Ae. albopictus, the magnitude of difference in survival between infected and uninfected mosquitoes was lower, with no significant difference in the case of GA-2 (Figure 2C). Overall, we found that both mosquito species had higher survival when infected by GA-2 compared to other parasite isolates (Figure 2C). However, contrary to expectation (see Figure 1) there was no overall difference in survival to EIP between mosquitoes infected with drug-susceptible (i.e., GA-2 and Missouri) vs. drug-resistant (i.e., Metairie and Yazoo) parasite isolates.

\subsection{Vectorial Capacity}

We found a higher vectorial capacity for Ae. aegypti infected by either GA-2 or Yazoo $D$. immitis (one drug-susceptible and one drug-resistant isolate, respectively) when compared to Ae. aegypti infected by Missouri or Metairie isolates. In the case of Ae. albopictus, we found that mosquitoes infected with Metairie and Yazoo (drug-resistant) isolates had higher vectorial capacity compared to GA-2 and Missouri (the drug-susceptible) isolates. However, this difference was only significant in the case of GA-2 and Metairie. Overall, our data indicated that the most important factor affecting vectorial capacity seems to be the D. immitis isolate, with the Metairie isolate-infected Ae. albopictus having very low vector efficiency compared to the Liverpool Blackeye isolate of Ae. aegypti (Figure 2B).

The structural equation model (SEM) revealed distinct paths affecting vectorial capacity (Figure 3A). Specifically, we found that establishment load positively affected vector efficiency (Figure 3B; $\beta \pm \mathrm{SE}=0.623 \pm 0.167 ; \mathrm{F}=13.788 ; \mathrm{DF}=20.239, p=0.001$ ), and in turn vectorial efficiency negatively affects survival to EIP (Figure 3C; $\beta \pm \mathrm{SE}=-0.619 \pm 0.167$; $\mathrm{F}=13.660 ; \mathrm{DF}=20.030, p=0.001)$. Importantly, we found that vectorial capacity was negatively affected by establishment load (Figure $3 \mathrm{D} ; \beta \pm \mathrm{SE}=-0.135 \pm 0.057 ; \mathrm{F}=5.606$; $\mathrm{DF}=18.091, p=0.029$ ), positively affected by vector efficiency (Figure $3 \mathrm{E} ; \beta \pm \mathrm{SE}=1.257 \pm$ $0.070 ; \mathrm{F}=322.795 ; \mathrm{DF}=18.238 ; p<0.001$ ) and positively affected survival to EIP (Figure 3F; $\beta \pm \mathrm{SE}=0.392 \pm 0.057 ; \mathrm{F}=44.472 ; \mathrm{DF}=19.012 ; p<0.001)$. Taking into consideration both direct and indirect effects of the variables affecting vectorial capacity (Figure 3G) we found significant positive total effects of establishment load [ $\beta_{\mathrm{TOT}}=0.275 ; 95 \%$ confidence interval (CI): $0.037,0.467]$, vector efficiency $\left(\beta_{\mathrm{TOT}}=0.609\right.$; $\mathrm{CI}$ : $\left.0.526,0.700\right)$ and survival to $\operatorname{EIP}\left(\beta_{\mathrm{TOT}}=0.304\right.$; CI: 0.219, 0.383). 
A

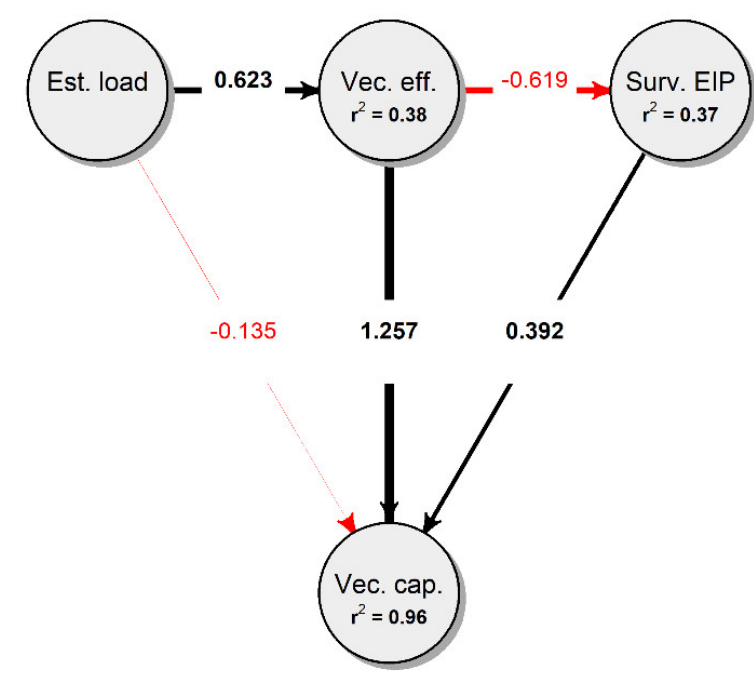

D

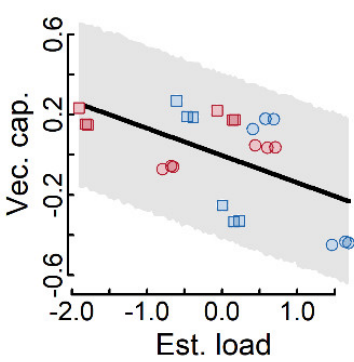

$\mathbf{E}$

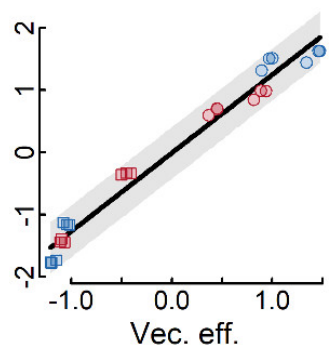

B

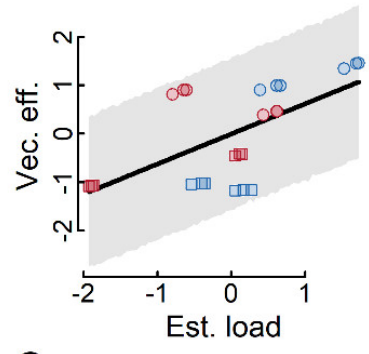

C

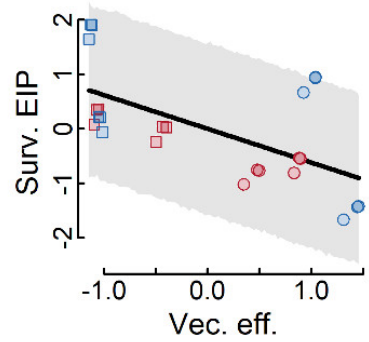

$\mathbf{F}$

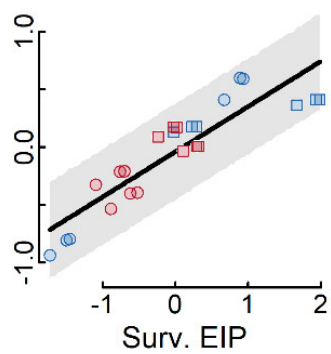

G

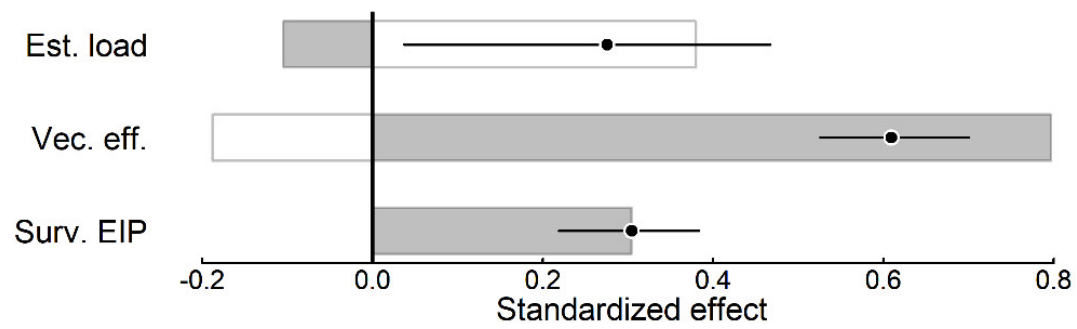

Figure 3. Causal pathways driving D. immitis vectorial capacity in Ae. mosquitoes. (A) The final structural equation model for vectorial capacity (Vect. Cap.) showing the direct and indirect effects of establishment load (Est. load), vector efficiency (Vect. Eff.) and survival to EIP (Surv. EIP). In the path diagram, circles indicate variables (with model $\mathrm{r} 2$ values, if applicable). Arrows indicate significant positive (black lines) or negative (red lines) relationships, with standardized coefficients indicated in bold. (B-F) Partial residual plots of the predictor ( $\mathrm{x}$ axis) and response (y axis) variables associated with all significant SEM paths. Individual points represent values obtained for each experimental replicate for Ae. aegypti (circles) and Ae. albopictus (squares) mosquitoes infected by either parasite isolates that were drug susceptible (GA-2 and Missouri; blue symbols) or drug resistant (Metairie and Yazoo; red symbols) with respective 95\% confidence intervals (CIs; gray bands). (G) Indirect (white bars), direct (gray bars) and total (circles) effects of establishment load, vector efficiency and survival to EIP on vectorial capacity. Error bars are 95\% CIs for the estimated total effects.

\section{Discussion}

The increase in anthelmintic resistance is a global issue that has critical implications for the current and future efficacy of preventatives and treatments for parasitic diseases of hu- 
mans and animals [19,25-27]. Investigating biological differences between drug-susceptible and drug-resistant parasites and their vectors should provide a better understanding of transmission dynamics and the evolutionary processes involved in the maintenance and spread [11,28] of drug resistance. Such knowledge could facilitate the development of novel treatments and further our understanding of mechanisms of anthelmintic resistance. We found that parasite isolates differed in terms of their initial establishment load, despite the fact that we standardized the $\mathrm{mf}$ concentration of each isolate in the blood to $5 \mathrm{mf} / \mu \mathrm{L}$, and that these isolates had differential effects on mosquito survival (see Figure 2). However, while mosquito mortalities for both Ae. aegypti and Ae. albopictus were species dependent, there was no discernable effect of drug-resistant or drug-susceptible status. Additionally, both the $D$. immitis isolate and Ae. spp. significantly impacted vector efficiency (the proportion of parasites developing to the infective stage at EIP). According to the evolutionary school of thought, we would expect that drug-resistant parasites would have lower fitness than drug-susceptible ones when drug-selective pressures are removed [29]. However, while we found strong parasite isolate and mosquito species interactions on vector efficiency, we found no evidence that the fitness of drug-resistant heartworm is reduced in the mosquito vector.

Drug resistance can have potential life history trade offs that influence parasite transmission. The Yazoo isolate of $D$. immitis exemplified a reduction in initial establishment load (Figure 2A) versus other D. immitis isolates. Interestingly, such reductions in initial establishment loads could potentially prove beneficial for reaching infective stages within the mosquito (Figure 2B). Intermediate infection doses maximize vector competence of D. immitis [14], which could provide an explanation for how a significant proportion of Yazoo L3s developed in comparison to the other parasite isolates. Another possible explanation to differential L3 development among D. immitis isolates is mosquito immunity pathways could play an important role in parasite establishment and development to transmissible stages [30]. However, more experiments are required to clarify whether the initial infective parasite dose of different $D$. immitis isolates has an impact on the development and survival of L3s, and vector parameters.

Our a priori prediction was that drug resistance would consistently reduce establishment load and increase vector efficiency (Figure 1). If the effects of reduced establishment load outweighed the effects of increased vector efficiency, we expected a lower infection intensity for drug-resistant vs. drug-susceptible parasites, and vice versa. In turn, we expected that infection intensity would be negatively correlated with survival to EIP, and thus with vectorial capacity (Figure 1). Thus, based on our theoretical model, we predicted that drug resistance could either increase or decrease vectorial capacity (Figure 1), based on the relative effects of drug resistance on establishment load vs. vector efficiency (Figure 1). However, our observed data revealed idiosyncratic patterns associated with the drugresistant and drug-susceptible isolates, with apparently no clear association between drug resistance and vectorial capacity (Figure 2D). This finding has critical implications for the spread of filarial drug resistance in natural populations because our data indicate that mosquitoes do not necessarily reduce the transmission efficacy of drug-resistant parasites, unlike in the case of relatively benign parasites such as Plasmodium [28,31].

While our data do not support our a priori expectations for the effects of drug resistance on vectorial capacity, they do provide a strong statistical model to better understand the underlying drivers affecting vectorial capacity for filarial parasites. Specifically, our SEM analyses revealed that establishment load had a slight negative direct effect on vectorial capacity (Figure 3G). However, this direct negative effect was overshadowed by a strong positive and indirect effect mediated primarily by the positive effect of establishment load on vector efficiency (Figure 3B). One important caveat of our study design was that mosquitoes were only exposed to a single parasite dose $(5 \mathrm{mf} / \mu \mathrm{L})$. Thus, in our case the positive association between establishment load and vector efficiency likely indicates underlying fitness differences between the parasite isolates. Thus, parasites that had low ability to establish infection initially also had a low likelihood of developing to infective 
L3 once they established infection. However, previous research has shown that the initial infection dose negatively affects vectorial capacity [14]. Thus, future research will need to test how alterations in parasite dose affects establishment load and vector efficiency. Interestingly, while our SEM model revealed a strong positive effect of vector efficiency on vectorial capacity (Figure 3E), there was significant, albeit weaker, negative effect of vector efficiency on vectorial capacity mediated by the negative effect of vector efficiency on survival to EIP (Figure 3C). Finally, as expected we found a strong positive effect of survival to EIP on vectorial capacity (Figure $3 F$ ).

In conclusion, when $D$. immitis infects the vector, the selective pressure of drug treatment is removed for the parasite, and this would be expected to allow drug-susceptible isolates of $D$. immitis to have the comparative advantage in the vector. We were interested in determining whether the development of drug resistance also impacted transmission dynamics by altering vectorial capacity. However, the data did not indicate a consistent pattern for drug-susceptible vs. drug-resistant isolates of $D$. immitis on vectorial capacity (Figure 2) as suggested by our predictive model (Figure 1). Thus, the data presented here indicate that overall patterns of vectorial capacity are potentially more complicated than what a categorization of drug-resistant and drug-susceptible parasites can discriminate, with a stronger effect due to mosquito species, compared to parasite isolates. However, despite the idiosyncratic patterns between the parasite isolates, we were able to elucidate strong causal relationships between the variables affecting vectorial capacity. Specifically, our data demonstrate that in the case of $D$. immitis, transmission risk is primarily driven by a positive indirect effect of establishment load on vectorial capacity, as well as positive direct effects of vector efficiency and survival to EIP on vectorial capacity. Thus, the spread of drug-resistant parasites in the vertebrate population is unlikely to be mitigated by reduced fitness in the mosquito vector.

\section{Materials and Methods}

\subsection{Simulation Model}

To generate quantitative expectations, we simulated the joint effects of establishment risk (i.e., the proportion of microfilariae that initially establish infection in the mosquito) and vector efficiency (i.e., proportion of microfilariae that establish infection that develop to L3 larvae) using the program R 4.0.0 (R Foundation for Statistical computing). We also simulated the probability of survival to the EIP (i.e., 14 days for $D$. immitis) assuming a parasite load-specific mortality using a common logistic function. We calculated vectorial capacity as the product of the three variables simulated. In other words, vectorial capacity is the proportion of microfilariae establishing infection that develop to L3 larvae in mosquitoes surviving to EIP [14]. To simulate the effects of drug resistance on vectorial capacity, we assumed that drug resistance would reduce microfilariae fitness in a drug-free environment (i.e., the mosquito). Thus, we simulated the effects of a reduction of $25 \%$ and $75 \%$ in establishment risk of the drug-resistant vs. drug-susceptible (i.e., reference) isolates. Additionally, we expected lower pathogenicity of the drug-resistant vs. drug-susceptible isolates, and thus expected higher vector efficiency in the latter vs. the former. We thus simulated the effects of a $25 \%$ and $75 \%$ increase in vector efficiency of the drug-resistant vs. drug-susceptible (i.e., reference) isolates. We plotted the combined effects of establishment risk, vector efficiency and survival to the EIP on vectorial capacity using a two-dimensional heatmap (as implemented in the $\mathrm{R}$ package raster). Further, we predicted the specific expectations for the nine possible outcomes based on all the factorial combinations associated with a $0 \%, 25 \%$ and $75 \%$ reduction in establishment risk, and a $0 \%, 25 \%$ and $75 \%$ increase in vector efficiency of the drug-resistant isolate compared to the drug-susceptible isolate (which was maintained at the reference value for establishment risk and vector efficiency).

\subsection{Experimental Procedures}

One hundred female Ae. aegypti and 60 female Ae. albopictus mosquitoes were allocated into separate cages per treatment: uninfected, Missouri, GA2, Metairie, and Yazoo. 
The mosquitoes were blood fed using a two-chamber inverted glass jacketed membrane feeder (see [14] for details). Briefly, adult female mosquitoes were removed with a mouth aspirator from each cage and placed into a new blood feeding cage. The blood feeding cages had their sugar removed the day prior and water removed at least $4 \mathrm{~h}$ prior to blood feeding.

To prepare the blood for feeding mosquitoes, we first estimated the microfilaremia in each infected blood sample by taking two $5 \mathrm{uL}$ aliquots from each sample and counting microfilariae under a compound microscope at $10 \times$ magnification. After counts were determined, all infected blood was diluted to a uniform count of $5 \mathrm{mf} / \mathrm{uL}$ concentration with uninfected blood. We then added $10 \mathrm{uL}$ of $100 \mathrm{mM}$ ATP (adenosine- $5^{\prime}$-triphosphate disodium salt, MP Biomedicals, Santa Ana, CA, USA) to each $1 \mathrm{~mL}$ of each blood sample as a phagostimulant (see [32] for details) prepared for blood feeders. We dispensed $200 \mathrm{uL}$ aliquots of blood (infected or uninfected as per treatment) into the blood feeders and mosquitoes fed for approximately two hours or until repletion. During feeding, the blood was kept at a constant temperature of $40{ }^{\circ} \mathrm{C}$ by circulating hot water through the external chamber of each feeder (see [14] for details). Ae. aegypti and Ae. albopictus were blood fed the same prepared blood within each treatment (i.e., uninfected or infected with the four D. immitis isolates).

The mosquitoes were blood fed following previously mentioned protocols; see above. Blood feeders were removed after feeding and replaced with water and sugar cubes. Afterwards, unfed mosquitoes were removed from the cages. On day five, Ae. albopictus mosquitoes were moved into new cages per treatment with a moist paper towel lined cup to allow oviposition. These mosquitoes oviposited for three days and then were moved to new cages per treatment, and maintained until the $D$. immitis microfilariae developed into the infective L3 stage (i.e., the extrinsic incubation period; EIP), which takes approximately 14 days for Ae. aegypti [17] and 13 days for Ae. albopictus [14].

During and/or after the experiment, the following data were collected with respect to three major parameters: establishment load, vector efficacy and survival to EIP. As mentioned above, we had specific expectations for each of the three variables (see Section 4.1). Thus, we used a power analysis to estimate the required sample size of mosquitoes to detect an effect size equivalent to a $20 \%$ change in the response variable as estimated from drug-resistant vs. drug-susceptible (reference category) parasites. We carried out all power analyses by generating simulated data using the sim.glmm function implemented in the $\mathrm{R}$ package GLMMMISC [33], fitting the appropriate statistical model for the response variable (see Section 4.4), and calculating power as the proportion of 1000 simulated datasets for which a significant effect was detected. Below we describe the procedures related to each of the three major parameters we focus on for this study.

\subsubsection{Establishment Load}

Immediately after blood feeding (1-2 h), we collected a subset of mosquitoes, and these mosquitoes were dissected to determine the observed number of microfilariae (mf) in the mid gut and Malpighian tubules, and thus calculate the initial establishment load. All experiments were performed in triplicate. For the establishment load analyses, we targeted a sample size of 15 mosquitoes ( 5 from each replicate) per parasite isolate and vector. Our power analyses indicated that this target sample size would give us a power of 0.818 to detect an effect size equivalent to a $20 \%$ reduction in establishment load in mosquitoes infected with drug-resistant vs. drug-susceptible parasites.

\subsubsection{Vector Efficiency}

To estimate vector efficiency, mosquitoes surviving past the EIP (14 days) were collected and dissected to quantify parasite load and characterize the stages of the different parasites detected. In the case of Ae. aegypti, we dissected all individuals that survived past the EIP and the experiment was terminated at day 17 post-feeding. In the case of Ae. albopictus we dissected all individuals that died after EIP, and to ensure sufficient sample size 
the experiment was terminated at day 21 post-feeding. During dissection, mosquitoes were categorized by the date of death or was killed, species of mosquito, replicate, and parasite treatment. D. immitis were categorized by life stage (mf, L1, L2, or L3) and counted for each dissection. Mosquito dissection and identification of parasite stages followed protocols described earlier [14]. All experiments were performed in triplicate. For the vector efficiency analyses, we targeted a sample size of 45 mosquitoes (15 from each replicate) per parasite isolate per vector. Our power analyses indicated that the target sample size (45 mosquitoes per parasite isolate and vector) would give us a power of 0.900 to detect an effect size equivalent to a $20 \%$ increase in vector efficiency in mosquitoes infected with drug-resistant vs. drug-susceptible parasites.

\subsubsection{Survival to EIP}

To calculate the proportion of mosquitoes surviving to the EIP, we recorded mosquito mortality daily after blood feeding. As mentioned previously, mosquito mortality was monitored for 17 and 21 days, in the case of Ae. aegypti and Ae. albopictus, respectively. At the end of the experimental period, all remaining mosquitoes were killed and considered to be right-censored data. All experiments were performed in triplicate. For the survival analyses, we targeted an initial population size of 135 blood fed female mosquitoes (45 from each replicate) per parasite isolate. Our power analyses indicated that this target sample size would give us a power of 0.820 to detect an effect size equivalent to a $20 \%$ increased probability of survival to EIP in mosquitoes infected with drug-resistant vs. drug-susceptible parasites.

\subsection{Study System}

This study focused on evaluating the vectorial capacity of mosquitoes infected with drug-resistant and drug-susceptible isolates of D. immitis, a mosquito-transmitted filarial parasite that primarily infects dogs and other canids. The D. immitis life cycle starts when the adult female releases microfilaria ( $\mathrm{mf}$ ) into the blood stream of the vertebrate host, usually a dog, which are subsequently taken up by a mosquito during blood feeding [16]. Microfilariae in the blood meal migrate to the Malpighian tubules, develop into first-stage larvae, and molt twice to reach their infective stage (L3) in the Malpighian tubule cells [15]. Once D. immitis reach their L3 stage, they migrate from the Malpighian tubules to the proboscis of the mosquito to be available for when the mosquito takes a blood meal, by entering the exit wound. The time D. immitis takes to develop to the L3 stage and reach the head of the proboscis is the extrinsic incubation period (EIP), approximately 14 days for Ae. aegypti [17] and 13 days for A. albopictus [14].

The D. immitis isolates that were chosen include two drug-resistant isolates MET.1 + .2 (2014) and YZO.B.1 + .2 (2013) (hereafter Metairie and Yazoo, respectively) and two drugsusceptible isolates MSO.1 (2000) and GAII.1.2t.3.4 (2013) (hereafter Missouri and Georgia-2, respectively). Detailed descriptions of these isolates have been described elsewhere [24]. The drug-resistant and drug-susceptible isolates of $D$. immitis were obtained from the Filariasis Research Reagent Resource Center and Kaplan laboratory at the University of Georgia.

The vectors used in this investigation were the yellow fever mosquito (Ae. aegypti) Liverpool Blackeye strain, and the Asian tiger mosquito (Ae. albopictus). Ae. aegypti and A. albopictus are both natural vectors of D. immitis in North America [13]. Ae. aegypti, populations used were established from eggs originally obtained from the Filariasis Research Reagent Resource Center [34], a long laboratory line highly susceptible to filarial parasites [35]. Ae. albopictus, wild type population, were obtained from Louisiana, $29^{\circ} 59^{\prime} 2.63^{\prime \prime}$ $\mathrm{N}$ and $90^{\circ} 7^{\prime} 0.36^{\prime \prime} \mathrm{W}$, and blood fed until repletion, subsequently placed into an insectary at UGA Savannah River Ecology Laboratory. Establishment of the laboratory line from natural populations followed [14]. All experiments described here were undertaken using F3 generation adult mosquitoes, for Ae. albopictus. Mosquitoes were maintained under 
standard insectary conditions: $27^{\circ} \mathrm{C}, 80 \pm 5 \%$ relative humidity, and a $12: 12 \mathrm{~h}$ light diurnal cycle (see [14] for details).

This vector-parasite combination is an ideal model system to study the effects of the mosquito vector on the spread of drug-resistant parasite isolates for several reasons: (a) it is a natural mosquito vector combination [16]; (b) it is logistically easy to work with due to ease of propagation and high infection susceptibility [17]; (c) the system is associated with strong co-evolutionary dynamics because the parasite likely places a large selective pressure on the mosquito due to the high mortality associated with infection $[14,36]$, and in turn the mosquito places a high selection pressure on the parasite, since parasites cannot be transmitted by dead mosquitoes [37]; (d) most importantly, there has been a well-documented and recent rise in drug resistance in D. immitis against macrocyclic lactones anthelmintics in the US [19], which has raised serious concerns about the future efficacy of these chemotherapeutic agents [20-22]. Drug resistance in D. immitis has been suspected for more than 15 years [38] and has been confirmed as a problem for over a decade $[19,39,40]$. The increasing incidence of resistance is a critical concern because the control of $D$. immitis depends almost exclusively on prophylactic treatment with macrocyclic lactone anthelmintic drugs [22].

\subsection{Data Analyses}

All statistical analyses were carried out using R 4.0.0. Analyses were primarily carried out using generalized linear mixed effects regression (GLMER) as implemented in the $\mathrm{R}$ package LME4 [41]. Regression results were modeled using least square means as implemented in the R package EMMEANS [42].

\subsubsection{Establishment Load}

Establishment load was defined as the initial number of $\mathrm{mf}$ per mosquito immediately after infection. We used a GLMER with a negative binomial error distribution (and log link) to model the initial $\mathrm{mf}$ dose as a linear effect of parasite isolate (Metairie, Yazoo, Missouri, GA2), as implemented in the R package LME4 [41]. We included replicate and status (i.e., died or censored) as random factors.

\subsubsection{Vector Efficiency}

Vector efficiency was defined as the proportion of developed L3 to the total number of parasites per mosquito surviving the extrinsic incubation period. We used a GLMER with a negative binomial error distribution (and log link) to model the total number of L3 parasites as a linear effect of parasite isolate (Metairie, Yazoo, Missouri, GA2). To control for the effects of establishment load, we included the initial $\mathrm{mf}$ dose as a model offset term. All models included replicate and status (i.e., died or censored) as random factors.

\subsubsection{Survival to EIP}

The probability of a mosquito surviving to EIP was modeled using a GLMER with a binomial error distribution. The status of the individual mosquito (i.e., dead or alive) was used as the dependent variable, and parasite isolate (Metairie, Yazoo, Missouri, GA2) as the independent variable. All models included replicate and status (i.e., died or censored) as random factors.

\subsubsection{Vectorial Capacity}

We estimated overall vectorial capacity as the joint probability of surviving to EIP and the risk of having L3 in mosquitoes surviving to EIP, following the approach described earlier [14]. Briefly, to calculate overall vectorial capacity we used a zero-inflated negative binomial regression approach and multiplied the estimated vector efficiency (which also controlled for effects of establishment load) with the probability of survival to EIP (see models above). We estimated the standard errors of this measure of vectorial capacity using parametric bootstrap (as implemented in the R package LME4). 
Finally, to obtain a better understanding of the causal relationships among the three major variables affecting vectorial capacity (i.e., establishment load, vector efficiency and survival to EIP), we constructed a structural equation model (SEM) ([43]) as implemented in the R package PIECEWISESEM ([44]). The initial model included three equations: (i) vector efficiency establishment load; (ii) survival to EIP establishment load + vector efficiency; (iii) vectorial capacity establishment load + vector efficiency + survival to EIP. We considered replicate a random effect in all models. All the SEM equations were modeled using linear mixed effects models (as implemented in LME4) after z-transformed all continuous variables to obtain standardized model coefficients. To test for potential non-linear effects between the variables we also included quadratic terms of all independent variables in the equations detailed above. We obtained the final SEM by sequentially dropping variables if dropping the variable from a specific model reduced the overall Akaike Information Criterion (AIC) of the SEM model ([45]), and if the removed equation was not considered to be a significant missing path ([44]). Final model acceptance was based on the Fisher's $C$ statistic, with a model being accepted if the associated $p$-value $>0.05$ ([43]). We also calculated the direct, indirect and total effects for each response variables in our final SEM model using the R package SEMEFF ([46]). The final path diagram was plotted using the $\mathrm{R}$ package DIAGRAM ([47]), and the strength of specific paths was assessed visually using partial residual plots using the R package VISREG ([48]).

Author Contributions: Conceptualization, E.N. and G.D.; methodology, E.N., C.C.E., P.D.J.C., R.M.K. and G.D.; formal analysis, E.N. and G.D.; investigation, E.N.; resources, R.M.K.; data curation, G.D.; writing—original draft preparation, E.N.; writing—review and editing, E.N., C.C.E., P.D.J.C., R.M.K. and G.D.; visualization, supervision, project administration and funding acquisition, G.D. All authors have read and agreed to the published version of the manuscript.

Funding: This research was funded by the U.S. Department of Energy Financial Assistance Award no. DE-EM0004391 to the University of Georgia Research Foundation.

Acknowledgments: We thank Andrew Moorhead and Erika Burkeman (Filariasis Research Reagent Resource Center) for providing expertise and resources. We would like to thank three anonymous reviewers for constructive comments on a previous draft of the manuscript. We thank Spencer Hills and colleagues at SREL for help and support.

Conflicts of Interest: The authors declare no conflict of interest. The funders had no role in the design of the study; in the collection, analyses, or interpretation of data; in the writing of the manuscript, or in the decision to publish the results.

\section{References}

1. WHO. Global Vector Control Response 2017-2030; World Health Organization: Geneva, Switzerland, 2017.

2. Bhatt, S.; Weiss, D.J.; Cameron, E.; Bisanzio, D.; Mappin, B.; Dalrymple, U.; Battle, K.E.; Moyes, C.L.; Henry, A.; Eckhoff, P.A.; et al. The Effect of Malaria Control on Plasmodium falciparum in Africa between 2000 and 2015. Nature 2015, 526, 207. [CrossRef] [PubMed]

3. Cibulskis, R.E.; Alonso, P.; Aponte, J.; Aregawi, M.; Barrette, A.; Bergeron, L.; Fergus, C.A.; Knox, T.; Lynch, M.; Patouillard, E.; et al. Malaria: Global progress 2000-2015 and Future Challenges. Infect. Dis. Poverty 2016, 5. [CrossRef] [PubMed]

4. Cromwell, E.A.; Schmidt, C.A.; Kwong, K.T.; Pigott, D.M.; Mupfasoni, D.; Biswas, G.; Shirude, S.; Hill, E.; Donkers, K.M.; Abdoli, A.; et al. The Global Distribution of Lymphatic Filariasis, 2000-2018: A Geospatial Analysis. Lancet Glob. Health 2020, 8, e1186-e1194. [CrossRef]

5. Conrad, M.D.; Rosenthal, P.J. Antimalarial Drug Resistance in Africa: The Calm before the Storm? Lancet Infect. Dis. 2019, 19, E338-E351. [CrossRef]

6. Churcher, T.; Chanda, E.; Coetzee, M.; Davenport, M.; Diabate, A.; Djimde, A.; Dondorp, A.M.; Donnelly, M.; Hemingway, J.; Huijben, S.; et al. malERA: An Updated Research Agenda for Insecticide and Drug Resistance in Malaria Elimination and Eradication. PLoS Med. 2017, 14. [CrossRef]

7. Misra-Bhattacharya, S.; Shahab, M. Progress in the Treatment and Control of Lymphatic Filariasis. In Lymphatic Filariasis: Epidemiology, Treatment and Prevention-The Indian Perspective; Tyagi, B.K., Ed.; Springer: Singapore, 2018; pp. 47-58.

8. Long, G.H.; Graham, A.L. Consequences of Immunopathology for Pathogen Virulence Evolution and Public Health: Malaria as a Case Study. Evol. Appl. 2011, 4, 278-291. [CrossRef]

9. Eisenstein, M. The Riddle of Resistance. Nature 2017, 543, S50-S51. [CrossRef] 
10. Webster, J.P.; Gower, C.M.; Norton, A.J. Evolutionary Concepts in Predicting and Evaluating the Impact of Mass Chemotherapy Schistosomiasis Control Programmes on Parasites and their Hosts. Evol. Appl. 2008, 1, 66-83. [CrossRef]

11. Goodman, C.D.; Siregar, J.E.; Mollard, V.; Vega-Rodriguez, J.; Syafruddin, D.; Matsuoka, H.; Matsuzaki, M.; Toyama, T.; Sturm, A.; Cozijnsen, A.; et al. Parasites Resistant to the Antimalarial Atovaquone Fail to Transmit by Mosquitoes. Science 2016, 352, 349-353. [CrossRef]

12. Ferguson, H.M.; Read, A.F. Why is the Effect of Malaria Parasites on Mosquito Survival still Unresolved? Trends Parasitol. 2002, 18, 256-261. [CrossRef]

13. Erickson, S.M.; Xi, Z.; Mayhew, G.F.; Ramirez, J.L.; Aliota, M.T.; Christensen, B.M.; Dimopoulos, G. Mosquito Infection Responses to Developing Filarial Worms. PLoS Negl. Trop. Dis. 2009, 3, e529. [CrossRef] [PubMed]

14. Dharmarajan, G.; Walker, K.D.; Lehmann, T. Variation in Tolerance to Parasites Affects Vectorial Capacity of Natural Asian Tiger Mosquito Populations. Curr. Biol. 2019, 29, 3946-3953. [CrossRef] [PubMed]

15. McCall, J.W.; Genchi, C.; Kramer, L.H.; Guerrero, J.; Venco, L. Chapter 4 Heartworm Disease in Animals and Humans. In Advances in Parasitology; Academic Press: Cambridge, Massachusetts, UK, 2008; Volume 6610, pp. 193-285. [CrossRef]

16. Bowman, D.D.; Atkins, C.E. Heartworm Biology, Treatment, and Control. Vet. Clin. N. Am. Small Anim. Pract. 2009, 39, 1127-1158. [CrossRef] [PubMed]

17. McCall, J.W. The Role of Arthropods in the Development of Animal-Models for Filariasis Research. J. Ga. Entomol. Soc. 1981, $16,283-293$.

18. Bowman, D.; Little, S.E.; Lorentzen, L.; Shields, J.; Sullivan, M.P.; Carlin, E.P. Prevalence and Geographic Distribution of Dirofilaria immitis, Borrelia burgdorferi, Ehrlichia canis, and Anaplasma phagocytophilum in Dogs in the United States: Results of a National Clinic-based Serologic Survey. Vet. Parasitol. 2009, 160, 138-148. [CrossRef]

19. Wolstenholme, A.J.; Evans, C.C.; Jimenez, P.D.; Moorhead, A.R. The Emergence of Macrocyclic Lactone Resistance in the Canine Heartworm Dirofilaria immitis. Parasitology 2015, 142, 1249-1259. [CrossRef]

20. Geary, T.G.; Bourguinat, C.; Prichard, R.K. Evidence for Macrocyclic Lactone Anthelmintic Resistance in Dirofilaria immitis. Top. Companion Anim. Med. 2011, 26, 186-192. [CrossRef]

21. Lee, A.C.; Montgomery, S.P.; Theis, J.H.; Blagburn, B.L.; Eberhard, M.L. Public Health Issues Concerning the Widespread Distribution of Canine Heartworm Disease. Trends Parasitol. 2010, 26, 168-173. [CrossRef]

22. Bowman, D.D.; Mannella, C. Macrocyclic lactones and Dirofilaria immitis Microfilariae. Top. Companion Anim. Med. 2011, 26, 160-172. [CrossRef]

23. Berrafato, T.; Coates, R.; Reaves, B.J.; Kulke, D.; Wolstenholme, A.J. Macrocyclic Lactone Anthelmintic-induced Leukocyte Binding to Dirofilaria immitis Microfilariae: Influence of the Drug Resistance Status of the Parasite. Int. J. Parasitol. Drug Resist. 2019, 10, 45-50. [CrossRef]

24. Sanchez, J.; Dharmarajan, G.; George, M.M.; Pulaski, C.; Wolstenholme, A.J.; Gilleard, J.S.; Kaplan, R.M. Using Population Genetics to Examine Relationships of Dirofilaria immitis based on both Macrocyclic Lactone-Resistance Status and Geography. Vet. Parasitol. 2020, 283, 10. [CrossRef] [PubMed]

25. Bowman, D.D. Heartworms, macrocyclic lactones, and the specter of resistance to prevention in the United States. Parasites Vectors 2012, 5, 10. [CrossRef] [PubMed]

26. Ertabaklar, H.; Malatyali, E.; Ertug, S. Drug Resistance in Parasitic Diseases. Eur. J. 2020, 26, 1-5. [CrossRef]

27. Kaplan, R.M. Drug Resistance in Nematodes of Veterinary Importance: A Status Report. Trends Parasitol. 2004, 20, 477-481. [CrossRef] [PubMed]

28. Mharakurwa, S.; Sialumano, M.; Liu, K.; Scott, A.; Thuma, P. Selection for Chloroquine-sensitive Plasmodium falciparum by Wild Anopheles arabiensis in Southern Zambia. Malar. J. 2013, 12, 6. [CrossRef] [PubMed]

29. Mackinnon, M.J.; Marsh, K. The Selection Landscape of Malaria Parasites. Science 2010, 328, 866-871. [CrossRef]

30. Edgerton, E.B.; McCrea, A.R.; Berry, C.T.; Kwok, J.Y.; Thompson, L.K.; Watson, B.; Fuller, E.M.; Nolan, T.J.; Lok, J.B.; Povelones, M. Activation of Mosquito Immunity Blocks the Development of Transmission-stage Filarial Nematodes. Proc. Natl. Acad. Sci. USA 2020, 117, 3711-3717. [CrossRef]

31. Spence, P.J.; Jarra, W.; Levy, P.; Reid, A.J.; Chappell, L.; Brugat, T.; Sanders, M.; Berriman, M.; Langhorne, J. Vector Transmission Regulates Immune Control of Plasmodium Virulence. Nature 2013, 498, 228. [CrossRef]

32. Pitts, R.J. A Blood-free Protein Meal Supporting Oogenesis in the Asian Tiger Mosquito, Aedes albopictus (Skuse). J. Insect Physiol. 2014, 64, 1-6. [CrossRef]

33. Johnson, P. Miscellaneous Functions for GLMMs. 2016. Available online: https://github.com/pcdjohnson/GLMMmisc (accessed on 8 February 2020).

34. Michalski, M.L.; Griffiths, K.G.; Williams, S.A.; Kaplan, R.M.; Moorhead, A.R. The NIH-NIAID Filariasis Research Reagent Resource Center. PLoS Negl. Trop. Dis. 2011, 5, e1261. [CrossRef]

35. Buxton, B.A.; Mullen, G.R. Comparative Susceptibility of 4 Strains of Aedes aegypti (Diptera, Culicidae) to Infection with Dirofilaria immitis. J. Med. Entomol. 1981, 18, 434-440. [CrossRef]

36. Lai, C.H.; Tung, K.C.; Ooi, H.K.; Wang, J.S. Competence of Aedes albopictus and Culex quinquefasciatus as Vector of Dirofilaria immitis after Blood Meal with Different Microfilarial Density. Vet. Parasitol. 2000, 90, 231-237. [CrossRef]

37. Bartholomay, L.C.; Michel, K. Mosquito Immunobiology: The Intersection of Vector Health and Vector Competence. Annu. Rev. Entomol. 2018, 63, 145-167. [CrossRef] [PubMed] 
38. Hampshire, V.A. Evaluation of Efficacy of Heartworm Preventive Products at the FDA. Vet. Parasitol. 2005, 133, 191-195. [CrossRef]

39. Bourguinat, C.; Keller, K.; Bhan, A.; Peregrine, A.; Geary, T.; Prichard, R. Macrocyclic Lactone Resistance in Dirofilaria immitis. Vet. Parasitol. 2011, 181, 388-392. [CrossRef]

40. Pulaski, C.N.; Malone, J.B.; Bourguinat, C.; Prichard, R.; Geary, T.; Ward, D.; Klei, T.R.; Guidry, T.; Smith, G.; Delcambre, B.; et al. Establishment of Macrocyclic Lactone Resistant Dirofilaria immitis Isolates in Experimentally Infected Laboratory Dogs. Parasites Vectors 2014, 7, 9. [CrossRef]

41. Bates, D.; Mächler, M.; Bolker, B.; Walker, S. Fitting Linear Mixed-Effects Models Using lme4. J. Stat. Softw. 2015, 67, 48. [CrossRef]

42. Lenth, R.V. Least-Squares Means: The R Package lsmeans. J. Stat. Softw. 2016, 69, 33. [CrossRef]

43. Shipley, B. Cause and Correlation in Biology: A User's Guide to Path Analysis, Structural Equations and Causal Inference with R; Cambridge University Press: Cambridge, UK, 2016.

44. Lefcheck, J.S. PIECEWISESEM: Piecewise Structural Equation Modeling in R for Ecology, Evolution, and Systematics. Methods Ecol. Evol. 2016, 7, 573-579. [CrossRef]

45. Shipley, B. The AIC Model Selection Method Applied to Path Analytic Models Compared using a D-Separation Test. Ecology 2013, 94, 560-564. [CrossRef]

46. Murphy, M. Semeff: Automatic Calculation of Effects for Piecewise Structural Equation Models. 2020. Available online: https:/ /CRAN.R-project.org/package=semEff (accessed on 21 October 2020).

47. Soetaert, K. Diagram: Functions for Visualising Simple Graphs (Networks), Plotting Flow Diagrams. 2020. Available online: https: / /CRAN.R-project.org/package=diagram (accessed on 21 October 2020).

48. Breheny, P.; Burchett, W. Visualization of Regression Models Using visreg. $R$ J. 2017, 9, 56-71. [CrossRef] 